\title{
Influence of thermochemistry on Mach reflection in hypersonic flow
}

\author{
M. Sharma, J. M. Austin ${ }^{\dagger}$ and N. G. Glumac ${ }^{\ddagger}$ \\ University of Illinois at Urbana-Champaign, IL 61801
}

\section{Introduction}

Real gas thermochemistry can significantly impact the aerodynamics of hypersonic systems. For example, shock stand-off distance in front of a blunt body has been shown to depend on the degree of chemical dissociation. ${ }^{1}$ High temperature effects can also alter shock-shock interaction phenomena, but the degree of the modification and its consequences can be challenging to predict. Sanderson et al. ${ }^{2}$ experimentally investigated oblique shock impingment on a bow shock (Edney type IV configuration) in a flow with significant gas dissociation. Previous studies ${ }^{3-5}$ had suggested significant increase in heat transfer at jet impingement due to real gas effects, however, experiments showed no dependence of peak heat transfer rate on stagnation enthalpy. The influence of nonequilibrium gas chemistry on Mach and regular shock reflection has been investigated in a number of numerical studies. ${ }^{6-8}$ Burtschell et al. ${ }^{8}$ numerically investigated a wedge geometry located in a Mach 7 free stream, a setup similar to that used in the present experimental work. Mach stem height and hysteresis behavior was examined. Burtschell et al. found a strong dependence of transition angles, Mach stem height and location on the gas flow model. For a given wedge angle, the inclusion of real gas chemistry led to a significant decrease in Mach stem height. Chemical-vibration coupling, however, slightly increased the height of the Mach stem. Direct Monte-Carlo simulations of a shock reflection with and without real gas effects carried out by Gimelschein et al. ${ }^{6}$ also found a substantial effect on Mach stem height and transition angle. However, an experimental study in dissociating nitrogen and carbon dioxide, ionizing argon and frozen argon could detect no effect on the transition condition due to finite relaxation length at the conditions of the experiment. ${ }^{9}$

In the present work, we experimentally investigate a Mach reflection generated by two opposing wedges in a Mach 7.1 free stream. The main goal of this work is to determine directly what kinds of real gas effects occur behind a normal shock in a Mach reflection configuration for a previously selected run condition. Experiments are carried out in an expansion tube facility which is capable of simulating high enthalpy hypersonic flight conditions, and a significant degree of vibrational excitation and chemical dissociation are expected behind the normal shock.

In high enthalpy gas flows, emission spectroscopy can be used to characterize the test gas composition and thermodynamic state. As impulse facilities, expansion tubes produce a challenging experimental environment for probe measurements with issues such as short test times, high temperatures and velocities, and diaphragm fragmentation. ${ }^{10,11}$ The non-intrusive nature of spectroscopy makes it an attractive technique for determining flow field properties in impulse facilities. Spectrally resolved studies have been previously used as a means towards characterizing high-enthalpy run conditions. Work completed at the X1 and X2 superorbital expansion tube facilities used emission spectroscopy to measure electron number density behind a bow shock and to identify sources of visible radiation. ${ }^{12,13}$ Time-resolved spectral methods were used in the JX1 expansion tube facility to determine the useful test time. ${ }^{14}$ Using the CARS technique, temperature profiles were determined for a hypervelocity blunt body flow field using the T3 shock tunnel facility. ${ }^{15}$ Using the free piston shock tube/tunnel facility TCM2, laser spectroscopy was used for species identification and shock front temperature profile diagnostics ${ }^{16}$ and spontaneous Raman spectroscopy was used to analyze the self-luminosity of nitrogen hypersonic flows for varying enthalpy conditions. ${ }^{17}$

\footnotetext{
* Graduate Student, Department of Aerospace Engineering, University of Illinois, Member AIAA

$\dagger$ Assistant Professor, Department of Aerospace Engineering, University of Illinois, Senior Member AIAA

$\ddagger$ Professor, Department of Mechanical Science and Engineering, University of Illinois, Senior Member AIAA
} 
In the current experiments, asymmetric wedges are used to generate a Mach stem, with a free shear layer at each triple point. Imaged spectroscopic measurements behind the Mach stem are presented. The spectra confirms flow dissociation and verifies the appropriateness of a run condition which in the future is to be used towards investigating high-temperature effects upon shear layer structure in hypersonic flow.

\section{Experimental Setup}

The Hypervelocity Expansion Tube (HET) facility at the University of Illinois consists of three sections: a driver, driven and accelerator section, all with inner diameter of $152 \mathrm{~mm}$. The HET is capable of accessing high stagnation enthalpies (approximately $8-10 \mathrm{MJ} / \mathrm{kg}$ ) and a Mach number range of 3.0 to $7.1{ }^{18}$ and is therefore suitable for examining high temperature real gas effects in a hypersonic flow. In the present study, an air test gas, helium driver and accelerator gas run condition (labelled Air-1) with Mach number 7.1 is used. With air as the test gas, reasonably strong emission lines are expected in the ultra-violet portion of the spectrum.

In the current experiment, a Mach reflection is created by an asymmetric wedge configuration as shown in Fig. 1 with free shear layers generated from shock triple points. The Mach reflection configuration was chosen partly because the triple point shear layer separates considerably different streams: a relatively hot, subsonic stream behind the Mach stem, and a relatively cold, supersonic stream behind the oblique waves. Significant $\mathrm{O}_{2}$ dissociation and NO formation is calculated to occur in the subsonic stream. ${ }^{19}$ The vast majority of experimental shear layer work has utilized the splitter plate configuration. The current geometrical arrangement does not suffer from typical splitter plate complications such as leading edge effects upon flow self-similarity and turbulence criterion. Amongst the literature a few exceptions are present which also avoid such issues, ${ }^{20}$ however, all of these investigate compressibility effects and none were performed in high-enthalpy conditions. Asymmetric wedge configurations have been previously experimentally studied such as, Li et al., ${ }^{21}$ however, the emphasis has always historically been upon the regular-Mach reflection hysteresis phenomenon.

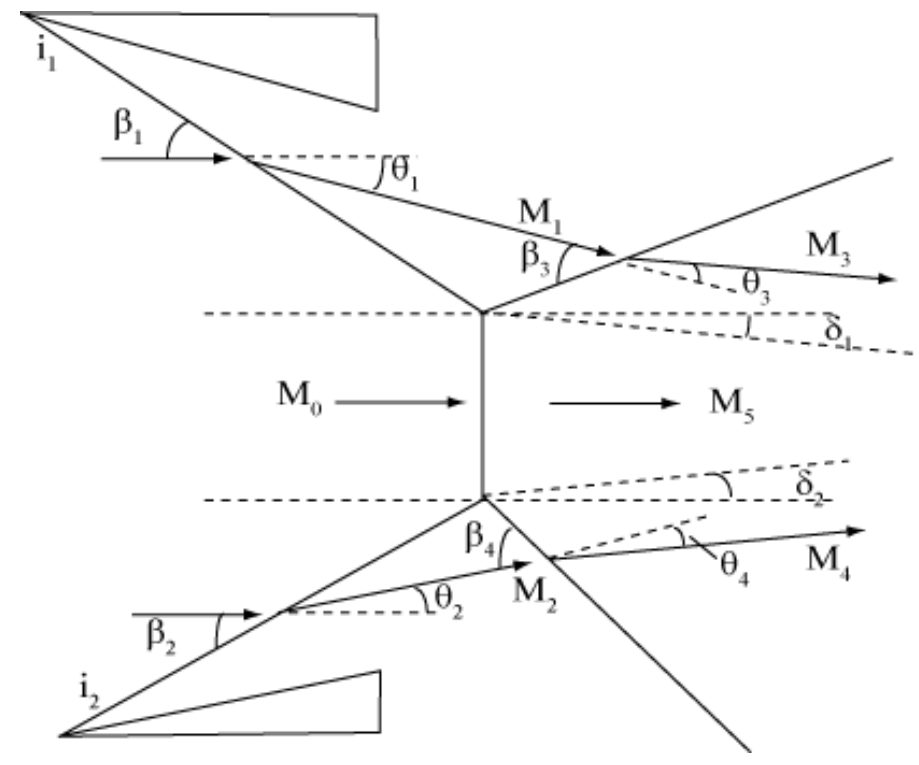

Figure 1. Schematic of an asymmetric Mach reflection

The experimental setup is shown in Fig. 2. A Xenon-437B Nanopulse system and PCO 1600 camera are used to obtain schlieren images. For spectroscopic measurements, emission light is collected and collimated through a window port and then condensed for a f/2 CP140 Jobin Yvon imaging spectrograph. The wavelength range of the spectrograph is $190-625 \mathrm{~nm}$ and the average dispersion at the exit is $17 \mathrm{~nm} / \mathrm{mm}$. Two different slit sizes of 25 and $50 \mu \mathrm{m}$ were used for which only the $50 \mu \mathrm{m}$ results are presented. A $6035 \mathrm{Hg}-\mathrm{Ar}$ pencil lamp was the calibration source. As the primary purpose of this study is species identification, no intensity calibration was required. Spectral lines were visualized using a Princeton Instruments PI-MAX MG:512SB intensified CCD camera system and a ST-133 camera controller. Both the spectrometer and 
schlieren acquistions were triggered by the transmitted shock arrival at the test section pitot probe which was located within the core flow $31.75 \mathrm{~mm}$ below the tube centerline. ${ }^{18}$ The pitot pressure also confirms schlieren and spectra acquisition during the test time. Driven section static pressure and test section pitot pressure, using PCB 113A26 dynamic pressure transducers, verify run condition flow properties and repeatability. Pressure data was recorded using a National Instruments data acquistion system, consisting of a PXI-1031 chassis, a BNC-2100 8 channel connector block and a 14-bit PXI-6133 3 MS/s simultaneous sampling multi-function data acquisition module. A full description of the facility, measurement capabilities and facility operation can be found in Dufrene et al. ${ }^{18}$

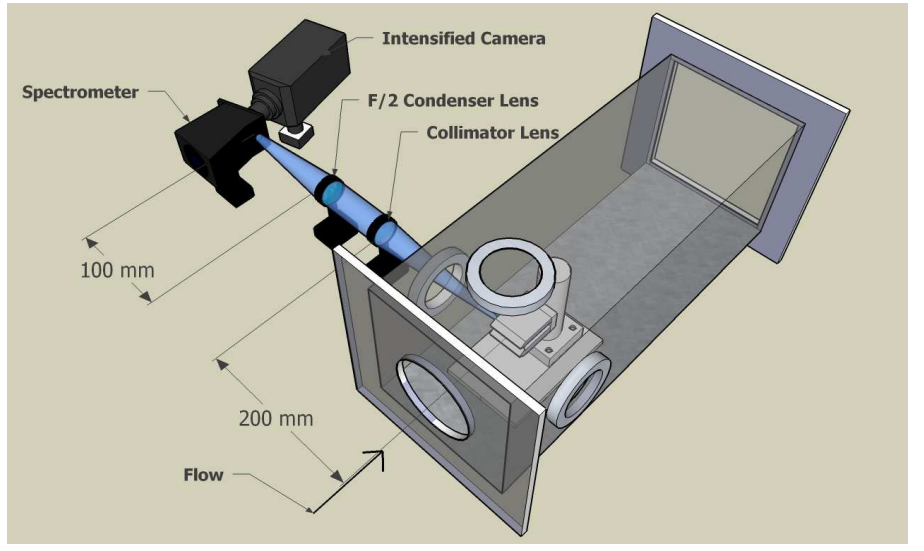

(a)

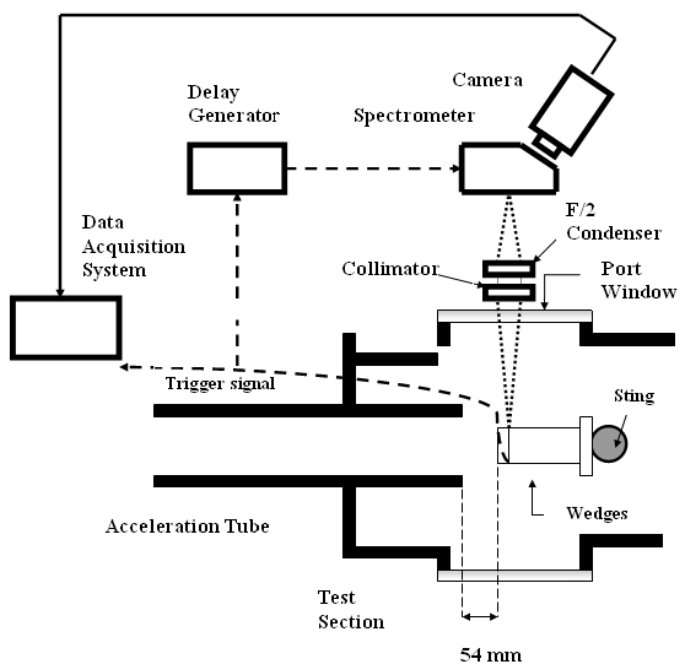

(b)

Figure 2. a) Three dimensional rendering of imaging spectroscopy collection system b) Schematic of experimental data acquisition setup

\section{Results}

To date, the Air-1 test condition has been characterized by driven and accelerator section shock speed measurements, test gas pitot pressure surveys, and the measurement of shock angles over cone and wedge geometries. ${ }^{18}$ Tables 1 and 2 present a summary of these results. Computational work has also been completed to verify the run condition. ${ }^{22}$ Theoretical prediction was obtained using inviscid perfect gas assumptions, for which 25 and 35 degree wedges were chosen as pressure-deflection polars predicted a Mach reflection for this configuration. In order to avoid three-dimensional effects, inlet aspect ratios of 1.25 and wedge aspect ratios of 5 were used. ${ }^{23}$

Schlieren visualization (as depicted in Fig. 3 a) has successfully established the presence and location of the Mach reflection in the Mach 7.1 free stream and was used for shock angle measurement. The center of the Mach stem is located $12.77 \mathrm{~mm}$ downstream of the wedge tip plane, is slightly off perpendicular and is $2.78 \mathrm{~mm}$ high. Highly resolved schlieren images with $4 \mathrm{~mm}$ field of view of the triple point shear (see Fig. 3 b) layer indicate that the triple point structure location is repeatable. The most marked difference between theory and experiment can be seen for the $\beta_{4}$ and $\delta_{2}$ measurement, which suggests the influence of non-equilibrium effects through the oblique shock reflection system. Preliminary computational results which compare the same run condition wedge configuration for non-equilibrium, equilibrium, perfect gas and frozen flow suggest that flow thermochemistry plays a significant role upon the Mach reflection shock angles, location of the Mach stem and even the type of the shock reflection (Mach or regular) system itself.

Calculated velocity, thermodynamic properties and species concentrations in the two shear layer streams for the lower (25 degree wedge) triple point shear layer are shown in Tables 3 and 4 . Values have been calculated assuming frozen flow directly downstream of the triple point, and assuming nonequilibrium flow at a distance $3 \mathrm{~cm}$ downstream of the triple point. The nonequilibrium calculation was carried out with 


\begin{tabular}{lcc}
\hline \hline & Theory & Exp. \\
\hline & & \\
Mach number & 7.29 & $\mathbf{7 . 1} \pm \mathbf{0 . 2}$ \\
$\mathrm{p}_{\text {pitot }, 7}, \mathrm{kPa}$ & 67 & $\mathbf{6 4}$ \\
$\mathrm{U}_{s}, \mathrm{~m} / \mathrm{s}$ & 2126 & $\mathbf{2 0 6 9} \pm \mathbf{4 3}$ \\
Test time, $\mu \mathrm{s}$ & 158 & $\mathbf{1 0 0}$ \\
$\mathrm{U}_{s, 7}, \mathrm{~m} / \mathrm{s}$ & 3998 & $\mathbf{4 1 4 8} \pm \mathbf{4 7}$ \\
\hline \hline
\end{tabular}

Table 1. Comparison between theoretical and experimentally measured flow parameters. State 7 denotes the test gas. $\mathbf{U}_{s}$ is the primary shock speed. Angle subscripts refer to Fig. 1.

\begin{tabular}{lcc}
\hline \hline Shock Angles & Theory & Exp. \\
\hline$\beta_{1}$, degrees & 46.69 & $\mathbf{4 4 . 4} \pm \mathbf{0 . 3}$ \\
$\beta_{2}$, degrees & 33.08 & $\mathbf{3 2 . 5} \pm \mathbf{0 . 3}$ \\
$\beta_{4}$, degrees & 40.83 & $\mathbf{4 5 . 0 5} \pm \mathbf{0 . 3}$ \\
$\delta_{2}$, degrees & -7.40 & $\mathbf{- 2 . 7 0} \pm \mathbf{0 . 3}$ \\
\hline \hline
\end{tabular}

Table 2. Comparison between theoretical and experimentally measured Mach reflection shock and shear layer angles. Angle subscripts refer to Fig. 1 .

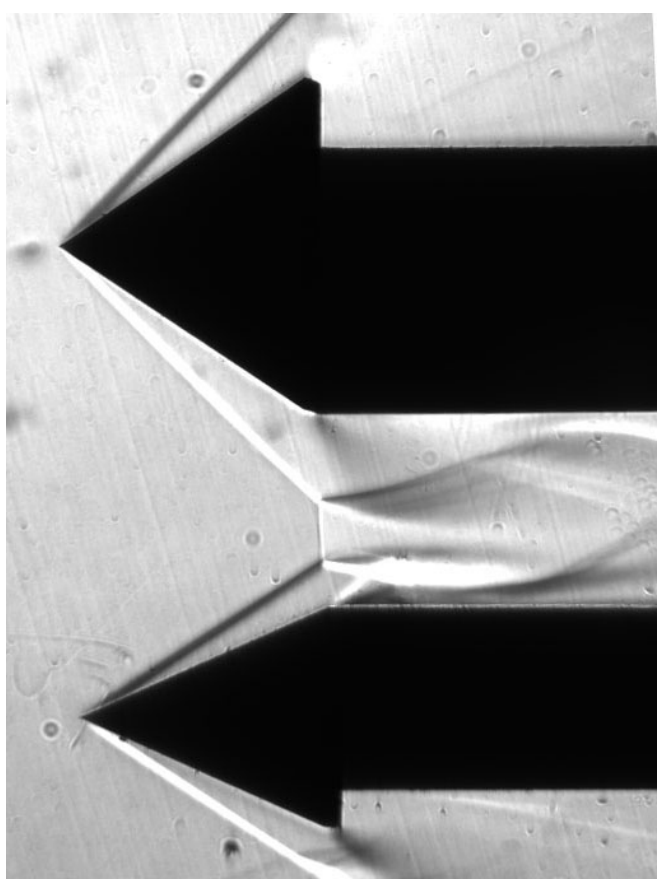

(a)
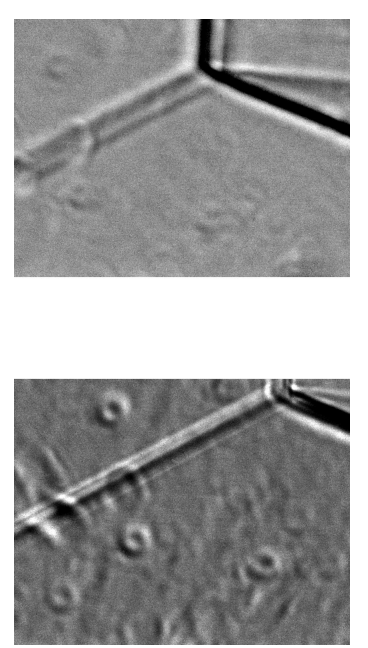

(b)

Figure 3. a) Schlieren image of Mach reflection for Mach 7.1 freestream flow (Air-1 run condition). Tip-to-tip wedge spacing is $25 \mathrm{~mm}$. b) Schlieren images of bottom triple point (associated with $25^{\circ}$ degree wedge) with a $4 \mathrm{~mm}$ field of view.

a FHO-FR model, which is a state-specific forced harmonic oscillator model for vibrational energy transfer rates, ${ }^{24}$ and assuming five species for air $\left(\mathrm{O}_{2}, \mathrm{~N}_{2}, \mathrm{NO}, \mathrm{O}\right.$ and $\left.\mathrm{N}\right) .{ }^{19}$ As Table 4 shows, the FHO-FR model calculations predict a temperature of $4600 \mathrm{~K}$ behind the Mach stem and $2500 \mathrm{~K}$ behind the oblique shock, ${ }^{19}$ and significant levels of dissociated $\mathrm{O}_{2}$, a portion of $\mathrm{N}$ and some NO.

Spectra were acquired using 25 and $50 \mu \mathrm{m}$ slits, with resolutions of $1.02 \mathrm{~nm}$ and $1.43 \mathrm{~nm}$ respectively. An example of the spectrum obtained is presented in Fig. 4 a) and b) for the $50 \mu \mathrm{m}$ slit. Spectra were obtained $33 \mathrm{~mm}$ downstream of the centre of the wedge tip plane, which based on schlieren images, places the imaging line $20.43 \mathrm{~mm}$ behind the Mach stem along the tube centerline. Imaging line spatial determination was obtained using a calibration lamp point source for which axial, transverse and vertical translation to within $1 \mathrm{~mm}$ spatial resolution was possible. An exposure time of $50 \mu \mathrm{s}$ was used, and pitot pressure traces confirm spectra acquistion during the test time. Reasonably strong signals from NO and ionized $\mathrm{N}_{2}$ can be seen. The strongest signal is due to $\mathrm{OH}$ emission, which is a result of the moisture within the test gas. Spectra obtained with the narrower $25 \mu \mathrm{m}$ slit displayed slightly finer scale structure than that resolved with the $50 \mu \mathrm{m}$ slit. However, as the exposure time is limited by the experimental test time and no signal amplification method 


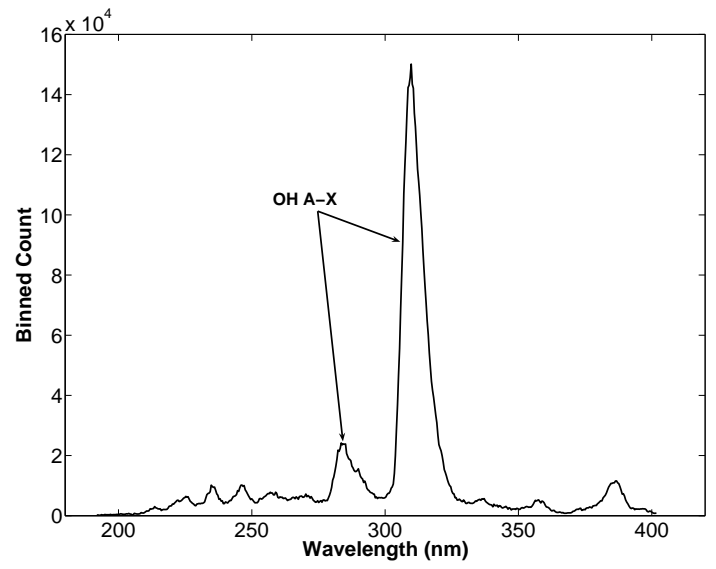

(a)

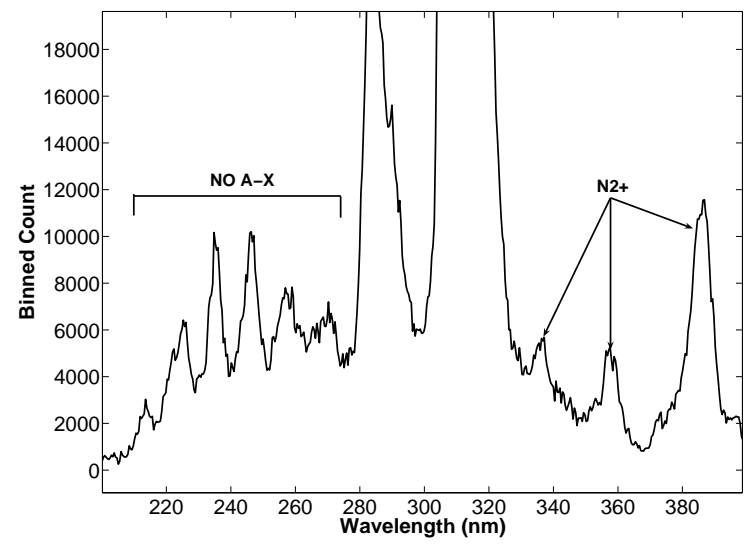

(b)

Figure 4. a) Full spectra for Air-1 run condition. Obtained at the center of the tube $20.43 \mathrm{~mm}$ downstream of Mach stem with a 50 micron slit and 50 microsecond exposure time. b) Zoomed in spectra of a)

\begin{tabular}{lrrrr}
\hline \hline & Frozen & $\left(M_{c}=0.683\right)$ & FHO-FR & $\left(M_{c}=0.884\right)$ \\
& MS & $\mathrm{I} / \mathrm{R}$ & MS & $\mathrm{I} / \mathrm{R}$ \\
\hline Velocity, $\mathrm{m} / \mathrm{s}$ & 726 & 2893 & 726 & 2893 \\
Density, $g / \mathrm{m}^{3}$ & 25 & 47 & 37 & 55 \\
Temperature, $K$ & 8329 & 4444 & 5231 & 3781 \\
\hline \hline
\end{tabular}

\begin{tabular}{lcc}
\hline \hline & Machstem & Oblique shocks \\
\hline $\mathrm{N}_{2}$ & 0.665 & 0.789 \\
$\mathrm{O}_{2}$ & $5.68 \times 10^{-3}$ & 0.210 \\
$\mathrm{NO}$ & 0.118 & $1.24 \times 10^{-4}$ \\
$\mathrm{~N}$ & $3.04 \times 10^{-3}$ & $2.21 \times 10^{-6}$ \\
$\mathrm{O}$ & 0.157 & $6.44 \times 10^{-4}$ \\
\hline \hline
\end{tabular}

Table 3. Shear layer flow properties at $25^{\circ}$ wedge. MS denotes Mach stem region and $I / R$ denotes oblique inci$\mathrm{dent} /$ reflection shock region. In column one, the convective Mach number $M_{c}$ is based on the frozen speed of sound; in column two on the the equilibrium speed of sound.

Table 4. Species mole fractions calculated $3 \mathrm{~cm}$ downstream of the lower triple point for Air-1 run condition.

has yet been implemented, the signal strength was roughly half that of the $50 \mu \mathrm{m}$ case. The finer spectral resolution did not provide a pronounced difference and improvements will be required to expose finer spectral structure, for example to differentiate the strong $297 \mathrm{~nm}$ O line. Greater signal or extra spatial resolution is needed to identify the presence of this species. The proximity of the strong $\mathrm{OH}$ emission could potentially overwhelm the $\mathrm{O}$ signal, however, progress towards eliminating the $\mathrm{OH}$ contamination is required before any further deductions can be made. The use of a spectrometer with greater spectral resolution, combined with the established $\mathrm{OH}$ A-X and NO A-X signal strength, should permit temperature diagnostic studies. The results confirm dissociating flow behind the Mach stem, which demonstrates that the flow field appears to be appropriate for high-temperature effect investigation.

\section{Conclusions}

Spectroscopic measurements demonstrate chemical dissociation behind normal and oblique shocks in a Mach reflection in hypervelocity flow. The Mach reflection was created using opposing wedges in a Mach 7.1 free stream. Experiments were performed in a newly constructed expansion tube facility. The Air-1 experimental condition used for the study has been previously verified by measurements of static and pitot pressure and shock speeds, ${ }^{18}$ whilst the Mach reflection configuration was verified via computational simulations. $^{22}$ Schlieren images show a $2.7 \mathrm{~mm}$ high Mach stem located $12.7 \mathrm{~mm}$ downstream of the wedge tips. Experimental measurements of the shock angles were compared with theoretical (perfect gas) predictions and significant departure of shear layer and reflected shock angles from perfect gas theory suggest that the thermochemistry has influenced the Mach reflection.

Emission spectroscopy was used to investigate gas dissociation. Spectra indicated the presence of ionized 
$\mathrm{N}_{2}$ and $\mathrm{NO}$ occuring behind the Mach stem, as predicted by nonequilibrium calculations. ${ }^{19}$ Good signal-tonoise levels were obtained, and significant contamination was found only due to $\mathrm{OH}$. Sufficient $\mathrm{NO}$ and $\mathrm{OH}$ signal was observed to indicate that with greater spatial resolution emission spectroscopy could be used as a temperature diagnostic. The presence of the $\mathrm{OH}$ could also possibly prove to be beneficial for other diagnostic techniques such as planar laser induced fluorescence. The current work also suggests the application of the method towards obtaining spectra at different vertical locations to provide information across the triple point shear layers. The Mach reflection configuration is part of a broader study to investigate thermochemical effects upon hypersonic shear layer structure.

\section{Acknowledgments}

This research was funded in part through AFOSR/MURI Grant FA9550-04-1-0425 with Dr. John Schmisseur as Technical Monitor.

\section{References}

${ }^{1}$ Wen, C. and Hornung, H., "Non-equilibrium dissociating flow over sphere," Journal of Fluid Mechanics, Vol. 299, 1995, pp. 389-405.

${ }^{2}$ Sanderson, S., Hornung, H., and Sturtevant, B., "The influence of non-equilibrium dissociation on the flow produced by shock impingement on a blunt body," Journal of Fluid Mechanics, Vol. 516, 2004, pp. 1-37.

${ }^{3}$ Edney, B., "Anomalous heat transfer and pressure distributions on blunt bodies at hypersonic speeds," FFA Report, Vol. 115, 1968.

${ }^{4}$ Hanneman, K., Brück, S., and Brenner, G., "Numerical simulation of reacting flows related to the HEG," 19th Int. Symp. Shock Waves, Marseille, 2 (eds. R. Brun and L.Z. Dumitrescu), 1993, pp. 251-256.

${ }^{5}$ Brück, S., "Investigation of shock-shock interactions in hypersonic reentry flows," Proc. 20th Int. Symp. Shock Waves, Pasadena, 1 (eds. B. Sturtevant, J. E. Shepherd, and H.G. Hornung), 1995, pp. 215-220.

${ }^{6}$ Gimelschein, S., Markelov, G., and Ivanov, M., "Relaxation and rarefaction effects on shock wave reflections," AIAA Paper 98-2819., 1998.

${ }^{7}$ Burtschell, Y., Cardoso, M., Zeitoun, D., and Abgrall, R., "Chemical nonequilibrium effects on RR $\leftrightarrow$ MR transition: numerical investigations," Proc. 21st Int. Symp. Shock Waves, Australia, 2 (ed. A.F.P.Houwing), 1995, pp. 825-830.

${ }^{8}$ Burtschell, Y., Zeitoun, D., and Ben-Dor, G., "Steady shock wave reflections in thermochemical nonequilibrium flows," Shock Waves, Vol. 11, 2000, pp. 15-21.

${ }^{9}$ Hornung, H., Oertel, H., and Sandeman, J., "Transition to Mach reflection of shock waves in steady and pseudosteady flow with and without relaxation," J. Fluid Mech., Vol. 90, 1979, pp. 541-560.

${ }^{10}$ Spurk, J. H., "Design, operation and preliminary results of the BRL expansion tube," Fourth Hypervelocity Technical Symposium, A.E.D.C, 1965, pp. 114-144.

${ }^{11}$ Jones, J., "Some performance characteristics of the LRC 3.75 inch pilot expansion tube using an unheated hydrogen driver," Fourth Hypervelocity Techniques Symposium, 1965, pp. 7-26.

${ }^{12}$ McIntyre, T., Bishop, A., Thomas, A., Wegener, M., and Rubinsztein-Dunlop, H., "Emission and holographic interferometry measurements in a superorbital expansion tube," AIAA Journal, Vol. 36, No. 6, 1998, pp. 1049-1054.

${ }^{13}$ McIntyre, T., Bishop, A., Thomas, A., Sasoh, A., and Rubinsztein-Dunlop, H., "Ionizing nitrogen and air flows ina superorbital expansion tube," AIAA Journal, Vol. 38, No. 9, 2000, pp. 1685-1691.

${ }^{14}$ Ramjuan, D., Sasoh, A., Ohnishi, Y., and Takayama, K., "Time-resolved spectroscopy in the shock layer of a blunt body placed in an expansion tube flow," 24th International Congress on High-Speed Photography and Photonics, Vol. $4183,2001$.

${ }^{15}$ Boyce, R., Pulford, D., Houwing, A., and Mundt, C., "Rotational and vibrational temperature measurements using CARS ina hypervelocity shock layer flow and comparisons with CFD calculations," Shcok Waves, Vol. 6, No. 1, 1995, pp. 41-51.

${ }^{16}$ Boubert, P., Chaiz, A., Chikhaoui, A., Robin, L., and Versich, P., "Aerodynamic calibration of TCM2 facility and study of a bow shock layer by emission and laser spectroscopy," Shock Waves, Vol. 11, 2002, pp. 341-351.

${ }^{17}$ Pilverdier, H., Brun, R., and Dumitrescu, M., "Emission and Raman spectroscopy measurements in hypersonic nitrogen flows," Journal of Thermophysics and Heat Transfer, Vol. 15, No. 4, 2001, pp. 484-490.

${ }^{18}$ Dufrene, A., Sharma, M., and Austin, J., "Design and characterization of a hypervelocity expansion tube facility," Journal of Propulsion and Power, Vol. 23, No. 6, 2007, pp. 1185-1193.

${ }^{19}$ Massa, L. and Austin, J., "Triple point shear layers in hypervelocity flow," submitted to Physics of Fluids A.

${ }^{20}$ Alvi, F., Krothapalli, A., and Washington, D., "Experimental study of a compressible countercurrent turbulent shear layer," AIAA Journal, Vol. 34, No. 4, 1996, pp. 728-735.

${ }^{21} \mathrm{Li}, \mathrm{H}$., Chpoun, A., and Ben-Dor, G., "Analytical and experimental investigations of the reflection of asymmetric shock waves in steady flows," Journal of Fluid Mechanics, Vol. 390, 1999, pp. 25-43.

${ }^{22}$ McGilvray, M., Austin, J., Sharma, M., Jacobs, P., and Morgan, R., "Diagnostic modelling of an expansion tube operating condition for a hypersonic shear layer experiment," Proceedings of the 16th Australasian Fluid Mechanics Conference, 2007.

${ }^{23}$ Skews, B., "Aspect ratio effects in wind tunnel studies of shock wave reflection transition," Shock Waves, Vol. 7, 1997, pp. $373-383$.

${ }^{24}$ Adamovich, I., "Three dimensional analytical model of vibrational energy transfer in molecule-molecule collisions," AIAA J., Vol. 39, 2001, pp. 10. 